\title{
An applied research to forecast the trend of air pollution in particular geometries of streets: the real case of urban canyon
}

\author{
F. Patania, A. Gagliano, F. Nocera, A. Galesi \& A. D’Amico \\ Energy and Environment Division of D.I.I.M., \\ Engineering Faculty of University of Catania, Italy
}

\begin{abstract}
High pollution levels have often been observed in urban street canyons due to the increased traffic emissions and reduced natural ventilation.

To investigate this phenomenon suitable microscale dispersion models must be used in order to give the right support to decision makers for air pollution control.

Within these streets, pedestrians, cyclists, drivers and residents are likely to be exposed to pollutant concentrations exceeding current air quality standards.

In this way people carried out an applied research choosing the "V. Giuffrida" street of Catania as a test canyon because this street shows both geometric features typical of an urban canyon and a lot of car traffic flowing during all the day.

In the first step the authors carried out preliminary and suitable studies about street and surrounding buildings geometries, meteoclimatic local data, intensity of traffic flow in different hours of day and so on.

In the second step a computational model of the street canyon has been developed taking into account all blocks of buildings on each side of the street according to the actual urban situation.

The computational model has been done using $\mathrm{K}-\varepsilon$ turbulence model and under three wind directions and velocity values characteristic of the area.

Finally, people carried out a campaign of concentrations measurements of tracer gas on suitable sections of the investigated street.

This paper intends to show:

- the methodologies and techniques to draw the investigation with the backing of a 3D computer code suitable for this use (Airpak - Fluent in our case).

- the particular flow-dynamic situations with formation of pollutant store zones.

- the influence of both buildings geometry and the presence of internal courtyard on pollution profiles.

- The distribution of gas concentrations along the facades of buildings flanking the street with reference to those zones which, overcoming the alarm limits of law, can cause detrimental effects on the health of the inhabitants.
\end{abstract}

Keywords: urban canyons, air pollution, urban traffic. 


\section{Generality}

Despite significant improvements in fuel and engine technology, urban environments are mostly dominated by traffic emissions. It is generally recognised that most of the substances directly emitted by vehicles in the ambient air or indirectly produced through photochemical reactions could represent a serious hazard for human health.

In urban environments and especially in those areas where population and traffic density are relatively high, human exposure to hazardous substances is expected to be significantly increased. This is often the case near busy traffic axis in city centres where urban topography and microclimate may contribute to condition of air stagnation giving rise to contamination hotspots. High pollution levels have been observed in "Urban Canyons", which is a term frequently used for urban streets bounded by buildings on both sides. Within these streets, pedestrians, cyclists, drivers and residents are likely to be exposed to pollutant concentrations exceeding current air quality standards.

The dimensions of an urban canyon are usually expressed by its aspect ratio, which is the height $(\mathrm{H})$ of the canyon divided by the width $(\mathrm{W})$. A canyon might be called regular, if it has an aspect ratio approximately equal to 1 and not big openings along the walls. An avenue canyon may have an aspect ratio below 0.5 , while a value of 2 may be representative of a deep canyon. Finally, the length (L) of the canyon usually expresses the road distance between two major intersections, subdividing street canyons into short $(\mathrm{L} / \mathrm{H} \approx 3)$, medium $(\mathrm{L} / \mathrm{H} \approx 5)$; and long canyons $(\mathrm{L} / \mathrm{H} \approx 7)$. Urban Canyons might be also classified in symmetric canyons if the buildings bounding the street have approximately the same height. On the contrary they may be classified asymmetric, if there are significant differences in building heights forming the canyon.

\section{The methodology of research}

The research has been carried out through the following steps:

1) Surveying of the area geometry by the available cartography

2) Surveying of the main traffic flows

3) Choice of Carbon Monoxide (CO) as the typical polluting gas from road traffic.

4) Campaign of measurements "in situ" of $\mathrm{CO}$ concentrations in eight points inside the canyon during some time periods of running traffic flow

5) Analysis of data registered by public environmental steady stations located near the urban canyon

6) Analysis of data acquired during the campaign of measurement

7) Application of AIRPAK computer code to forecast the trend of dispersion of exhausts along the canyon

8) Comparison between data measured and those forecasted or registered. 


\section{The investigated area}

The investigated urban canyon is located in the commercial centre of Catania city (V. Giuffrida street) as shown in figure 1. Along the whole course of this road there are heavy traffic flows both during day and nighttime. Geometrically the street, which presents the roadway ten meters wide, is continuously bordered in both sides by buildings that are between twelve and twenty meters high on average.

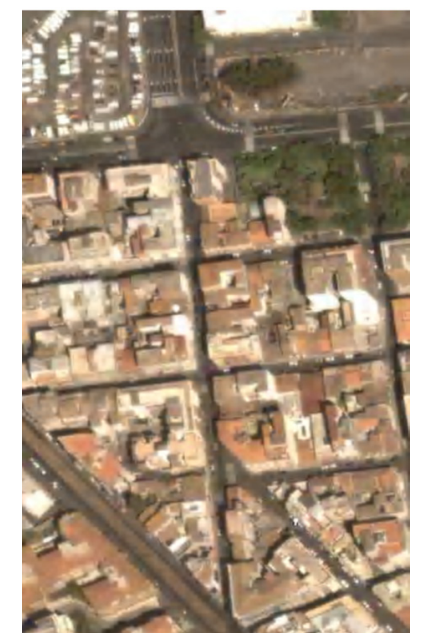

Figure 1: $\quad$ Investigated area.

People adopted Carbon Monoxide (CO) as tracer pollutant gas because of good chemical stability in urban atmosphere, easiness in the use of equipments of measurements. Moreover, $\mathrm{CO}$ is the typical gas emitted by vehicular traffic, as its concentration and the traffic load tend to have a similar trend of variation during the day.

Input data required by investigation are:

Values of car traffic flow

Features of circulating fleet of cars (class of power, kind of fuel, age of cars)

Emission factors (EF) of Carbon Monoxide

Mean velocity of car traffic flow

Fuel consumption of car as an unction of power class and relative discharge -rate of exhausts.

To monitor maximum traffic flows people subdivided the daytime in four periods of monitoring as shown in Table 1.

For each period two surveys of car flow (15 minutes for each survey) have been registered by video camera. The successive vision of registered video and the relative analysis took to data shown in figure 2 (referred to monitoring of total car traffic of V. Giuffrida street and R. Sanzio Street). 
Table 1: Daytime period.

\begin{tabular}{|ll|l|l|l|l|}
\cline { 3 - 6 } \multicolumn{1}{c|}{} & & hh:mm & hh:mm & hh:mm & hh:mm \\
\hline- & From & $07: 30$ & $10: 00$ & $13: 00$ & $18: 00$ \\
\hline- & To & $08: 30$ & $11: 00$ & $14: 00$ & $19: 00$ \\
\hline
\end{tabular}

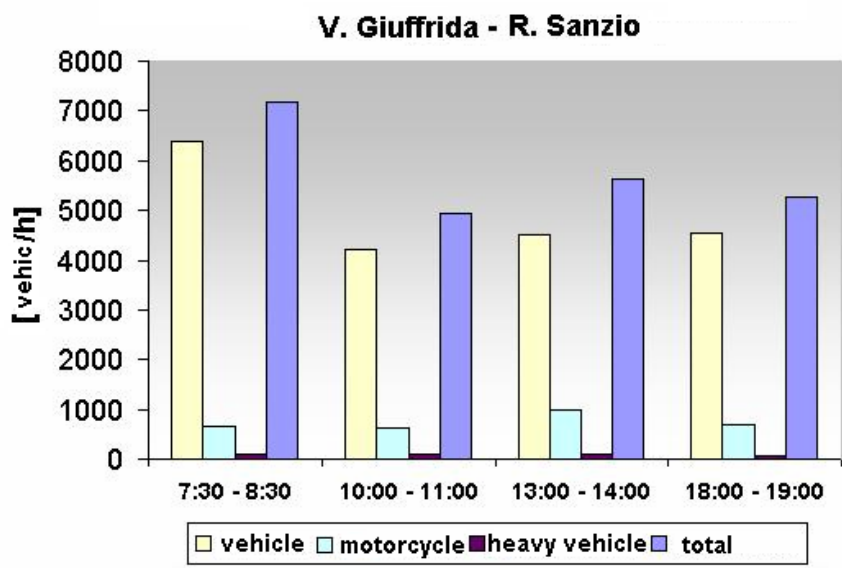

Figure 2: Traffic flow.

\section{The model of dispersion}

People used Airpak, which is a three-dimensional, steady, $\mathrm{k}-\varepsilon$ turbulence model to investigate the high Reynolds number skimming flow field of an urban canyon. The choice comes from the capability of Airpak to deal with complex shaped walls and other boundary conditions using flexible fine-scale grids.

Airpak is a CFD modelling based on the numerical solution of the governing fluid flow and dispersion equations which are derived from basic conservation and transport principles: the mass conservation (continuity) equation, the three momentum conservation (Navier-Stokes) equations in $\mathrm{x} ; \mathrm{y} ; \mathrm{z}$; the transport equation for pollutant concentration. The equations of state (obtained through the thermodynamic equilibrium assumption) and the Newtonian model of viscous stresses are also enlisted to close the system numerically, while initial and boundary conditions have to be specified to characterize external wind flows by the wind direction, wind speed, height above the ground, and description of the local terrain.

The code solves the Navier-Stokes equations for transport of mass, momentum, species, and energy when it calculates laminar flow with heat transfer. Additional transport equations are solved when the flow is turbulent or when radiative heat transfer is included.

The equation for conservation of mass, or continuity equation, can be written as follows: 


$$
\frac{\partial \rho}{\partial t}+\frac{\partial \rho}{\partial x_{i}}\left(\rho \cdot u_{i}\right)=0
$$

Transport of momentum in the $i^{\text {th }}$ direction in an inertial (non-accelerating) reference frame is described by

$$
\frac{\partial}{\partial t}\left(\rho \cdot u_{i}\right)+\frac{\partial}{\partial x_{j}}\left(\rho \cdot u_{i} \cdot u_{j}\right)=\frac{\partial p}{\partial x_{i}}+\frac{\partial \tau_{i j}}{\partial x_{j}}+\rho \cdot g_{i}+F_{i}
$$

where $\mathrm{p}$ is the static pressure, $\tau_{\mathrm{ij}}$ is the stress tensor, and $\rho g_{\mathrm{i}}$ is the gravitational body force in the $\mathrm{i}$ direction. $\mathrm{F}_{\mathrm{i}}$ contains other source terms that may arise from resistances, sources, etc. The energy conservation equation for a fluid region written in terms of sensible enthalpy $h$ is:

$$
\frac{\partial}{\partial t}(\rho \cdot h)+\frac{\partial}{\partial x_{i}}\left(\rho \cdot u_{i} \cdot h\right)=\frac{\partial}{\partial x_{i}}\left(k+k_{t}\right) \cdot \frac{\partial T}{\partial x_{i}}+S_{h}
$$

where $\mathrm{k}$ is the molecular conductivity, $\mathrm{k}_{\mathrm{t}}$ is the conductivity due to turbulent transport and the source term $\mathrm{S}_{\mathrm{h}}$ includes any volumetric heat sources you have defined. The species transport equations takes the following general form

$$
\frac{\partial}{\partial t}\left(\rho \cdot m_{i^{\prime}}\right)+\frac{\partial}{\partial x_{i}}\left(\rho \cdot u_{i} \cdot m_{i^{\prime}}\right)=-\frac{\partial}{\partial x_{i}} J_{i^{\prime} i}+S_{i^{\prime}}
$$

where $\mathrm{Si}^{\prime}$ is the rate of creation by addition from user-defined sources.

The code used the standard $k-\varepsilon$ model based on model transport equations for the turbulent kinetic energy $(\mathrm{k})$ and its dissipation rate $(\varepsilon)$. The model transport equation for $\mathrm{k}$ is derived from the exact equation, while the model transport equation for $\varepsilon$ is obtained using physical reasoning and bears little resemblance to its mathematically exact counterpart. The turbulent kinetic energy, $k$, and its rate of dissipation, $\varepsilon$, are obtained from the following transport equations:

$$
\begin{gathered}
\rho \frac{D k}{D t}=\frac{\partial}{\partial x_{i}}\left[\left(\mu+\frac{\mu_{t}}{\sigma_{k}}\right) \frac{\partial k}{\partial x_{i}}\right]+G_{k}+G_{b}-\rho \varepsilon \\
\rho \frac{D \varepsilon}{D t}=\frac{\partial}{\partial x_{i}}\left[\left(\mu+\frac{\mu_{t}}{\sigma_{\varepsilon}}\right) \frac{\partial \varepsilon}{\partial x_{i}}\right]+C_{1 \varepsilon} \cdot \frac{\varepsilon}{k} \cdot\left(G_{k}+C_{3 \varepsilon} G_{b}\right)-C_{2 \varepsilon} \rho \frac{\varepsilon^{2}}{k}
\end{gathered}
$$

In these equations, $G_{k}$ represents the generation of turbulent kinetic energy due to the mean velocity gradients, $G_{b}$ is the generation of turbulent kinetic energy due to buoyancy, $\mathrm{C}_{1 \varepsilon}, \mathrm{C}_{2 \varepsilon}$, and, $\mathrm{C}_{3 \varepsilon}$ are constants. $\sigma_{\mathrm{k}}$ and $\sigma_{\varepsilon}$ are the turbulent Prandtl numbers for $k$ and $\varepsilon$ respectively. 


\section{The simulation data}

As shown in figure 3 the chosen domain of calculus, in Cartesian coordinates has the following dimensions:

\begin{tabular}{|c|c|c|}
\hline X direction & Y direction & Z direction \\
\hline $700.0 \mathrm{~m}$ & $60.0 \mathrm{~m}$ & $700.0 \mathrm{~m}$ \\
\hline
\end{tabular}

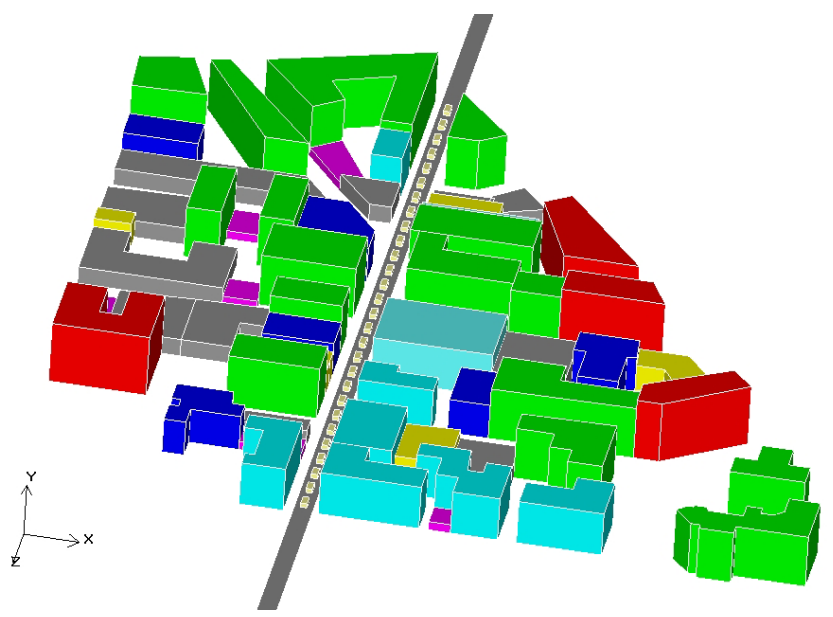

Figure 3: Buildings in 3D domain.

The grid of calculus becomes thicker and thicker inside the urban canyon and close to the ground level. The maximum dimension of the grid is $x=25.0, y=10 \mathrm{~m}$ and $\mathrm{z}=25 \mathrm{~m}$ on the Atmospheric Boundary Layer while the minimum dimension of the mesh is $\mathrm{x}=\mathrm{y}=\mathrm{z}=0.01 \mathrm{~m}$ on the exhausts pipe. People schematized the continuous linear source, typical of vehicular emission, as twenty-eight lined up point sources uniformly distributed along the axis of the canyon. The point sources show the following characteristics:

- Height of sources $\mathrm{h}=0.20 \mathrm{~m}$, that is about the position of exhausts pipe above the ground.

- Velocity of emission of exhausts (V=5.0m/s), coming from measurements by portable anemometer (TESTO 452).

- Velocity of exhausts has a vertical component $\left(i=5^{\circ}\right)$ to the aim to consider phenomena of turbulence produced by vehicles along the road.

People modelled terrain and buildings both by the available cartography at scale 1:2000 and by inspection carried out in the area. The ground has been considered flat because of the orography but people have chosen the roughness of boundary surfaces of street equal to $0.30 \mathrm{~m}$ while $1.50 \mathrm{~m}$ for the remaining ground.

To determine the meteoclimatic conditions associated to flows of traffic, people utilized both portable meteoclimatic station (Testo 452) placed at $1.5 \mathrm{~m}$ up 
the ground inside the Canyon and registered data coming from public meteoclimatic steady stations placed at about $10 \mathrm{~m}$ up the ground.

The simulations have been performed in order to verify the sensitivity and the reliability of the code and to forecast gaseous pollution according with meteorological data shown in table 2. Furthermore, every simulation has been done taking into account vehicles disposed in line and then to quincunxes

Table 2: $\quad$ Meteorological input data.

\begin{tabular}{|l|c|c|c|c|c|c|c|}
\hline Simulation number & 1 & 2 & 3 & 4 & 5 & 6 & 7 \\
\hline Wind direction & East & East & East & S-E & S-E & S-E & South \\
\hline Wind velocity $(\mathrm{m} / \mathrm{s})$ & 1.0 & 2.5 & 4.0 & 1.0 & 2.5 & 4.0 & 4.0 \\
\hline
\end{tabular}

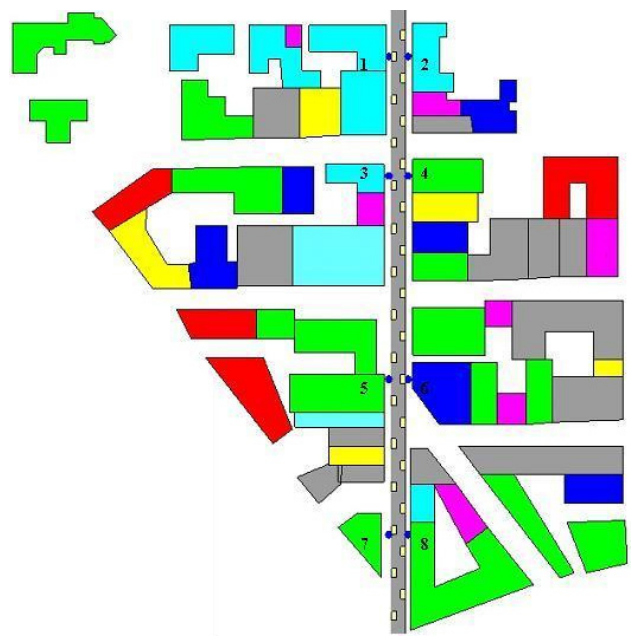

Figure 4: Points of measurement.

\section{Measurement in situ of $\mathrm{CO}$ concentration}

People carried out a simultaneous measurement campaign of $\mathrm{CO}$ concentrations to be able to successively compare data forecasted by the code and those coming from measurements. Measures have been done by means of a portable analyzer (catalytic bead technology - Multirae Plus PGM 50) at the same time of the measures of flows of traffic. Figure 4 briefly summarizes the position of each point of measure in investigated area.

\section{Results}

For direction of wind perpendicular to the canyon axis, people notices, according to theoretical studies:

- The air flow in the canyon can be seen as a secondary circulation feature driven by above roof imposed flow. If the wind speed out of the 
canyon is below $2.5 \mathrm{~m} / \mathrm{s}$, the coupling between the upper and secondary flow is lost and the relation between speed of wind above the roof and within the canyon is characterized by a considerable scattering.

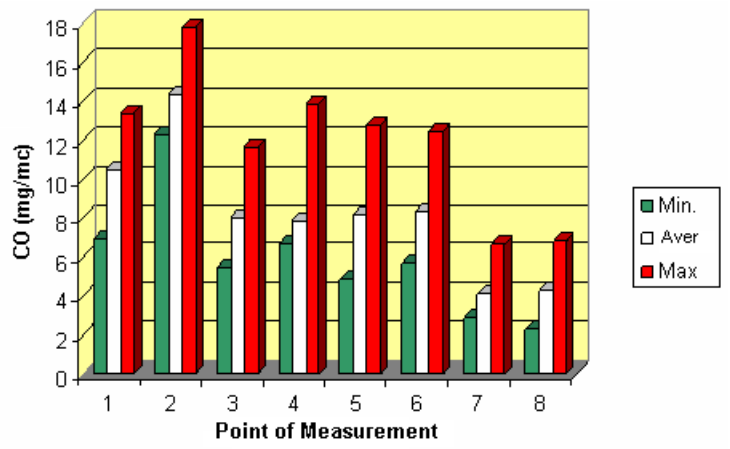

Figure 5: $\quad$ Exemplum of datasheet of measurement - 12 January 2005.
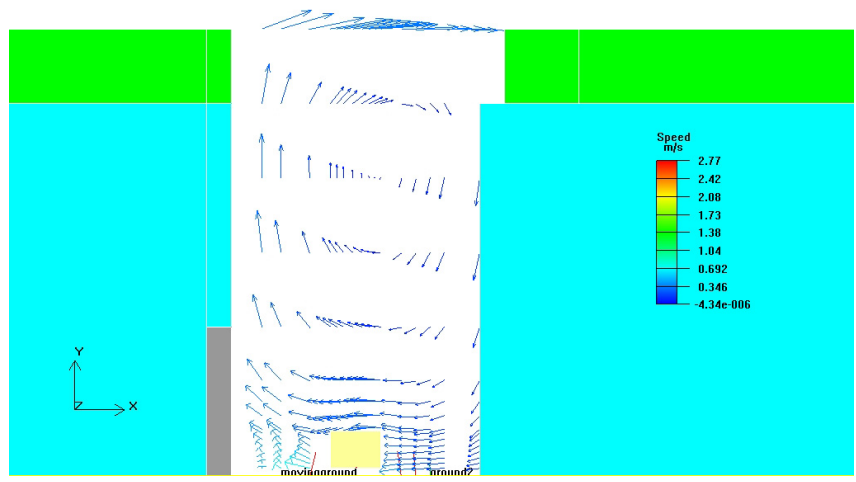

Figure 6: $\quad$ Flow pattern $-\mathrm{Pz}=60 \mathrm{~m}-\mathrm{Nz}=1-\mathrm{v}=1.0 \mathrm{~m} / \mathrm{s} \perp$ canyon.

- Regarding the direction of the vortex, it has to be expected that, as the vortex is driven by a downward transfer of momentum across the roof level shear zone, a flow normal to the canyon axis should create a vortex with the air near the ground flowing opposite to wind direction outside the canyon.

- $\quad$ People have found that high wind speeds $(>2.5 \mathrm{~m} / \mathrm{s})$ produce transverse and longitudinal stable whirls between buildings façade.

- The transverse stable whirls are originated by the impact of vertical component of the wind on the windward building façade while the longitudinal stable whirls are originated both by the buildings with different heights, flanking the street, and by transversal streets converging in the canyon, which create vortex circulation. 


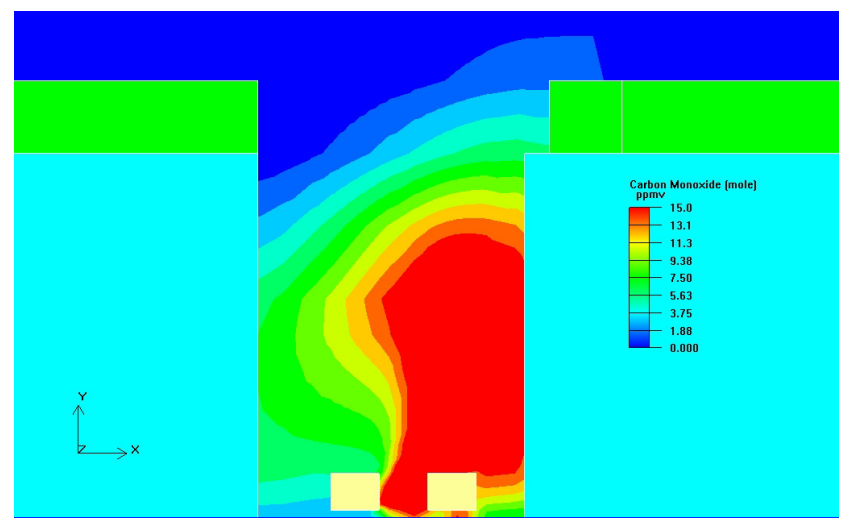

Figure 7: Exemplum of $\mathrm{CO}$ iso-concentration $\mathrm{Pz}=60 \mathrm{~m}-\mathrm{Nz}=1-\mathrm{v}=1.0 \mathrm{~m} / \mathrm{s} \perp$ canyon.

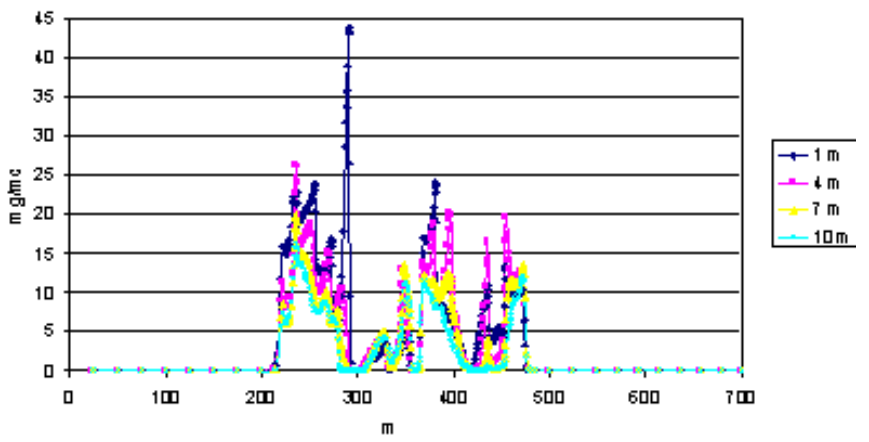

Figure 8: Exemplum of variation of $\mathrm{CO}$ concentration with the height in the leeward region of the canyon.

- In this case finite length canyon effects play an important role on the air flow distribution in the canyon. In fact the authors have found intermittent vortices on the buildings corner. These vortices are responsible for the mechanism of advection from the building corner to mid block creating a convergence zone in the mid block region of the canyon.

- Vertical velocities in the center of the vortex of the canyon have been measured to be close to zero.

Moreover people notices:

- an evident variation of direction of velocity near intersection with transversal streets that allow the inlet of air flow coming from zones outside canyon.

- the biggest value of pollutants concentration is forecasted close to the face of leeward buildings near the jets positions. 


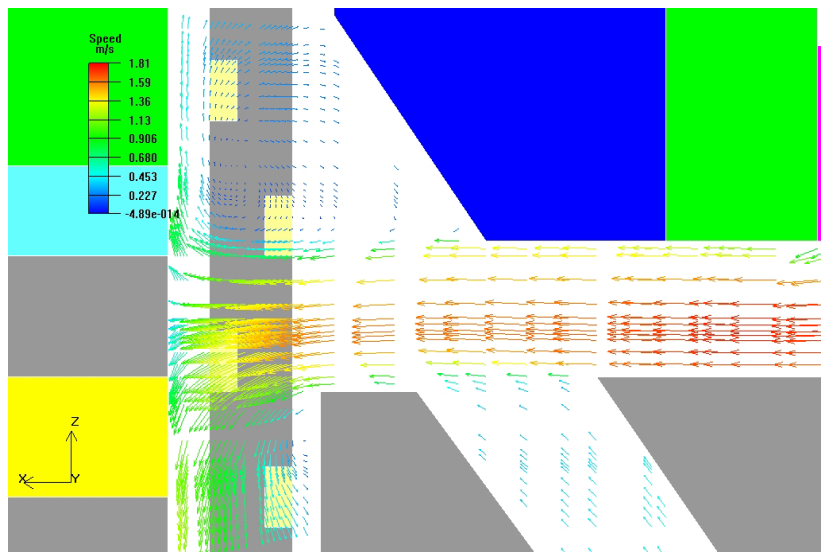

Figure 9: Exemplum of flow pattern $-\mathrm{Py}=3.00 \mathrm{~m}-\mathrm{Ny}=1-\mathrm{v}=2.5 \mathrm{~m} / \mathrm{s}$.

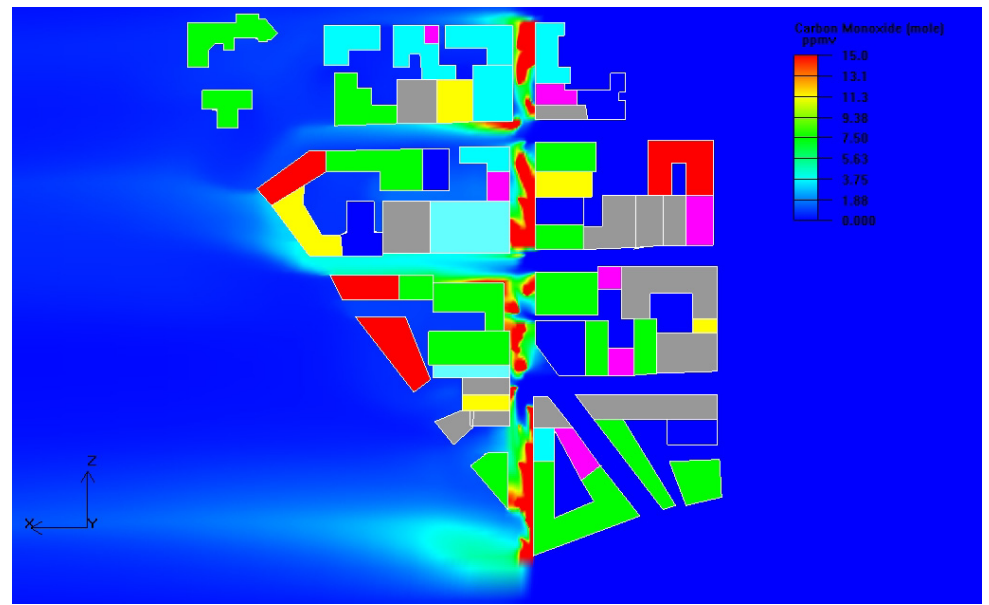

Figure 10: Exemplum of $\mathrm{CO}$ iso-concentration map $\mathrm{v}=1.00 \mathrm{~m} / \mathrm{s}$.

- $\quad$ the dispersions of pollutant gases inside the canyon is very small both in longitudinal and in cross directions. As far as longitudinal direction is concerned, this is due to a very negligible component of wind vector, whereas, in the cross direction, the air flow motion forces the pollution between the facades of building on both side.

- a very little component of wind velocity along the axis of canyon

- there is an accumulation of polluting concentrations on leeward side of building caused by buildings geometry, vortex circulation, transversal street and courtyards that supports the formation of depression zones.

- $\quad$ there are values of concentrations between 10 and $100 \mathrm{mg} / \mathrm{m}^{3}$ of CO.

- the concentration of $\mathrm{CO}$ for leeward façade is twice as high as for windward façade. 
By comparison between results coming from simulations and those coming both from experimental measurements in situ and from registration by steady station, it is possible to remark:

- the concentration values of $\mathrm{CO}$ coming from simulations are close to the concentration values coming from measurements in situ.

- the concentration values of $\mathrm{CO}$ measured in situ are three times or more greater than that registered by steady station of V. Giuffrida.

Table 3: Comparison between values of $\mathrm{CO}$ concentration with wind $1.20 \mathrm{~m} / \mathrm{s}$ direction East - date: 20/01/2005.

\begin{tabular}{|l|l|}
\hline Value of Concentration & $\begin{array}{c}\mathrm{CO} \\
\mathrm{mg} / \mathrm{m}^{3}\end{array}$ \\
\hline Forecasted & 7.00 \\
\hline Measured in Situ & 6.30 \\
\hline Registered by Steady Station & 1.20 \\
\hline
\end{tabular}

\section{Conclusions}

An experimental campaign of measurements and eighteen simulations have been performed to take into account different scenarios in function of intensity and direction of wind with reference to canyon axis, On the basis of the achieved results, it has been possible to point out that the fluid-dynamic profiles are able to maintain into the canyon gaseous polluting concentrations almost always superior to those prescribed by Italian Environmental Legislation. Only in the scenario showing the wind blowing with velocity of $4.00 \mathrm{~m} / \mathrm{s}$ and in direction parallel to street canyon, the value of concentration does not exceed law-limits. This last one scenario, to tell the truth, is very unusual since the velocity of wind during all the year in this canyon seldom comes up to $3.5 \mathrm{~m} / \mathrm{s}$ and the direction of the wind is variable during the day as it happens in all marine cities.

\section{References}

[1] S. J. Jeong, M. J. Andrews (2002). Application of the k-E turbulence model to the high Reynolds number skimming flow field of an urban street canyon. Atmospheric Environment Vol. 36, 1137-1145.

[2] Technical report No 49, COPERT III: Computer programme to calculate emissions from road transport Methodology and emission factors (Version 2.1) L. Ntziachristos and Z. Samaras ETC/AEM, November 2000.

[3] Oke, T.R., 1988. Street design and urban canopy lay and climate. Energy and Buildings 11, 103-113.

[4] F. Patania, A. Gagliano, F. Nocera Incompleteness of the urban traffic plan in preliminary studies of environmental impact: the real case of urban canyon" Urban Transport XI, Editors C.A. Brebbia. L.C. Wadhawa, WIT Press (U.K.). (2005) pp.417- 428. 
[5] F. Patania, A. Gagliano, F. Nocera, A. Galesi "An applied test to evaluate the use of fluid dynamic code as a predictive key of air pollution from urban road traffic" Urban Transport X, Editors C.A. Brebbia WIT Press (U.K.). (2004) pp.711-716.

[6] F. Patania, A. Gagliano, F. Nocera "Air pollution and urban plan of road traffic: experimental research about situations of environmental health hazard" - Urban Transport IX. Editors, C.A. Brebbia, L.J. Sucharov. WIT Press Southampton (U.K.). (2003) pp.253-262. 